\title{
Numerical simulations of vertical oscillations of a solar coronal loop
}

\author{
M. Selwa ${ }^{1}$, K. Murawski ${ }^{1}$, S. K. Solanki ${ }^{2}$, T. J. Wang ${ }^{2}$, and G. Tóth ${ }^{3}$ \\ ${ }^{1}$ Institute of Physics, UMCS, ul. Radziszewskiego 10, 20-031 Lublin, Poland \\ e-mail: mselwa@kft.umcs.lublin.pl \\ 2 Max-Planck-Institut für Sonnensystemforschung, Max-Planck-Str. 2, 37191 Katlenburg-Lindau, Germany \\ 3 Department of Atomic Physics, Loránd Eötvös University, Pázmány Péter sétány 1/A, Budapest 1117, Hungary
}

Received 23 March 2005 / Accepted 23 May 2005

\begin{abstract}
We consider the impulsive excitation of fast vertical kink standing waves in a solar coronal loop that is embedded in a potential arcade. The two-dimensional numerical model we implement includes the effects of field line curvature and nonlinearity on the excitation and damping of standing fast magnetosonic waves. The results of the numerical simulations reveal wave signatures which are characteristic of vertical loop oscillations seen in recent TRACE observational data.
\end{abstract}

Key words. Sun: oscillations - Sun: corona - magnetohydrodynamics (MHD)

\section{Introduction}

Solar magnetohydrodynamic (MHD) waves are an important diagnostic tool of the medium through which they propagate (Roberts at al. 1984; Roberts 2000, 2002; Nakariakov 2003; Klimchuk et al. 2004). They are also natural carriers of energy and probably transport some of the energy needed to heat the solar corona.

The last decade has seen the discovery of a variety of coronal loop oscillations and waves. Both propagating waves and various standing modes have been detected. These include transverse oscillations (i.e. oscillations of the loop perpendicular to the plane containing the unperturbed loop; Aschwanden et al. 1999), standing longitudinal slow modes (Wang et al. 2002), and most recently vertical oscillations (i.e. transverse oscillations within the plane containing the unperturbed loop; Wang \& Solanki 2004). Here we are interested in the vertical oscillations of a solar coronal loop. The observed loop (recorded by TRACE in active region 9893 at the northwest limb) expanded and contracted with an oscillation period of about $230 \mathrm{~s}$ after being triggered by a flare. The initial velocity of the loop was estimated as $\sim 130 \mathrm{~km} \mathrm{~s}^{-1}$ and the displacement amplitude of the loop apex as $7900 \mathrm{~km}$. The loop length was estimated as 300-400 Mm. An interesting feature of these oscillations is the evidence that they are compressible.

Oscillations that are presumably triggered by sudden energy release processes such as solar flares were discussed recently by Nakariakov \& Ofman (2001), Cooper et al. (2003), and Nakariakov et al. (2004). Terradas \& Ofman (2004) discussed the possibility that transverse waves in coronal loops can produce density enhancements at the apex. The mechanism involved was the ponderomotive force of standing MHD waves. Terradas et al. (2005) studied the excitation and damping of transversal coronal loop oscillations, using a simple line-tied one-dimensional model. Shergelashvili et al. (2005) demonstrated swing interaction between fast magnetosonic and Alfvén waves in an inhomogeneous medium. They showed that the fast magnetosonic waves propagating across an applied non-uniform magnetic field can parametrically amplify Alfvén waves propagating along the field, through the periodical variation of the Alfvén speed. Slow standing waves were discussed by Selwa et al. (2005) using parametric studies in the one-dimensional approximation. Fast standing waves were numerically simulated by Murawski et al. (2005a) who found high-order standing fast kink mode oscillations in a loop that is embedded in a weak magnetic environment. Murawski et al. (2005b) extended this work to the case of a general magnetic field neglecting gas pressure. They showed that impulsively triggered fast magnetosonic waves in a cold loop have periods which are compatible with both the period of the fast kink mode in the arcade and the observational data provided by TRACE. Transverse oscillations in solar coronal loops induced by propagating Alfvénic pulses were discussed by del Zanna et al. (2005).

In this paper we extend the models of Murawski et al. (2005a,b) by taking into account small but finite gas pressure effects. We aim to explain the recent observational findings by Wang \& Solanki (2004). We consider a two-dimensional curved magnetic field topology in a strongly magnetized coronal plasma.

The paper is organized as follows: the physical model and numerics are described in Sect. 2. The numerical results are 
presented in Sect. 3. This paper is concluded by a short summary of the main results in Sect. 4.

\section{Physical model and numerics}

Our model system is taken to be composed of a magnetized plasma with infinite electrical conductivity that behaves like an ideal gas with polytropic index $\gamma=5 / 3$. As we are interested in characteristic spatial scales lower than the pressure scale height in the solar corona, we neglect the effect of gravitational stratification. This assumption is not fully justified as the density scale height $H=46 \mathrm{Mm}$ for an isothermal loop with electron temperature $T_{\mathrm{e}}=1 \mathrm{MK}$ (Aschwanden 2002), while the loop height considered here is $h=70 \mathrm{Mm}$. Indeed, the effect of gravity is important in giving the plasma $\beta$ a realistic distribution with height.

With the above assumptions, the evolution of the system is modeled by the ideal MHD equations, which describe the conservative transport of mass, momentum, energy, and magnetic flux, viz.

$\frac{\partial \varrho}{\partial t}+\nabla \cdot(\varrho \boldsymbol{V})=0$,

$\frac{\partial(\varrho \boldsymbol{V})}{\partial t}+\nabla \cdot[(\varrho \boldsymbol{V}) \boldsymbol{V}]=-\nabla p+\frac{1}{\mu}(\nabla \times \boldsymbol{B}) \times \boldsymbol{B}$,

$\frac{\partial E}{\partial t}+\nabla \cdot\left[\left(E+p_{\mathrm{T}}\right) \boldsymbol{V}-\frac{\boldsymbol{B}(\boldsymbol{B} \cdot \boldsymbol{V})}{\mu}\right]=0$

$\frac{\partial \boldsymbol{B}}{\partial t}=\nabla \times(\boldsymbol{V} \times \boldsymbol{B})$,

$\nabla \cdot \boldsymbol{B}=0$.

Here $\varrho$ is the mass density, $\boldsymbol{V}$ is the flow velocity, $\boldsymbol{B}$ is the magnetic field with the magnetic permeability $\mu, p_{\mathrm{T}}$ denotes the total pressure given by the sum of the gas and magnetic pressures:

$p_{\mathrm{T}}=p+\frac{B^{2}}{2 \mu}$.

The plasma energy density $E$ is expressed by this pressure as

$E=\frac{\varrho V^{2}}{2}+\frac{p}{\gamma-1}+\frac{B^{2}}{2 \mu}$

\subsection{Equilibrium configuration}

We use the coronal arcade model that has been described by Oliver et al. (1998). In this model the coronal arcade is settled in a motionless environment $(\boldsymbol{V}=0)$. The assumption of a low plasma $\beta$ implies that the pressure gradient $\nabla p$ can be neglected in comparison to the Lorentz force, so that

$(\nabla \times \boldsymbol{B}) \times \boldsymbol{B}=0$.

We are interested in the case of a potential magnetic field with zero current,

$\nabla \times \boldsymbol{B}=0$.

We limit our discussion to a two-dimensional magnetically structured atmosphere for which the plasma quantities are independent of the spatial coordinate $y, \partial / \partial y=0$. We assume
Table 1. Scale and equilibrium parameters.

\begin{tabular}{cccccc}
\hline \hline$L[\mathrm{Mm}]$ & $\varrho_{0}\left[\frac{\mathrm{kg}}{\mathrm{m}^{3}}\right]$ & $V_{\mathrm{A} 0}\left[\frac{\mathrm{m}}{\mathrm{s}}\right]$ & $c_{\mathrm{s}}\left[\frac{\mathrm{m}}{\mathrm{s}}\right]$ & $B_{0}[G]$ & $T_{0}[\mathrm{MK}]$ \\
\hline 100 & $10^{-12}$ & $10^{6}$ & $10^{5}$ & 11 & 0.44 \\
\hline
\end{tabular}

that the unperturbed magnetic field is $\boldsymbol{B}=\left(B_{x}(x, z), 0, B_{z}(x, z)\right)$ and that $V_{y}=B_{y}=0$ for all time. In such a system we can express the equilibrium magnetic field in terms of a magnetic potential $\boldsymbol{A}=A \hat{\boldsymbol{y}}$ as

$\boldsymbol{B}=\nabla \times \boldsymbol{A}$,

where $\hat{\boldsymbol{y}}$ is a unit vector along the $y$-direction. Combining Eqs. (9) and (10) we obtain Laplace's equation, $\nabla^{2} A=0$, with solution

$A(x, z)=B_{0} \Lambda_{B} \cos \left(x / \Lambda_{B}\right) \mathrm{e}^{-z / \Lambda_{B}}$.

This method leads to the equilibrium magnetic field components

$B_{x}=B_{0} \cos \left(x / \Lambda_{B}\right) \mathrm{e}^{-z / \Lambda_{B}}$,

$B_{z}=-B_{0} \sin \left(x / \Lambda_{B}\right) \mathrm{e}^{-z / \Lambda_{B}}$.

Here $B_{0}$ is the magnetic field strength at the level $z=0$ and $\Lambda_{B}$ is the magnetic scale height. Requiring $B_{x}$ to vanish at $x / \Lambda_{B}=$ $\pm \pi / 2$, we find that

$\Lambda_{B}=\frac{2 L}{\pi}$

where $L$ denotes the half-width of the arcade.

For the potential magnetic arcade the equilibrium pressure $p_{0}$ has to be constant. This pressure can be evaluated from the reference sound speed $c_{\mathrm{s}}$ as

$p_{0}=\frac{1}{\gamma} \varrho_{0} c_{\mathrm{s}}^{2}$.

In a gravity-free atmosphere we can assume that the background mass density is constant, viz. $\varrho_{0}=$ const. Such a choice implies an isothermal atmosphere with constant sound speed and background temperature $T_{0}$. The field strength $B_{0}$ at the level $z=0$ is connected to the background density $\varrho_{0}$ through the Alfvén speed $V_{\mathrm{A} 0}$ as

$B_{0}^{2}=\mu \varrho_{0} V_{\mathrm{A} 0}^{2}$,

where $V_{\mathrm{A} 0}=$ denotes the Alfvén speed at the level $z=0$. Scale and equilibrium parameters introduced above are shown in Table 1.

We consider a "loop" that is embedded in the arcade such that its edges follow magnetic field lines ${ }^{1}$. We chose the mass density contrast $\varrho_{\mathrm{i}} / \varrho_{0}=10$ given by a step-function profile with $\varrho_{\mathrm{i}}$ corresponding to the internal mass density. This loop does not have a perfect circular shape, but its average radius

1 The "loop" is itself infinitely extended in the $y$ direction and thus strictly speaking also an arcade. However, we refer to it as a loop in order to distinguish it from the full magnetic field structure it is embedded in. 
and length can be estimated as $70 \mathrm{Mm}$ and $190 \mathrm{Mm}$, respectively. These values are similar to those of the loop observed by Wang \& Solanki (2004). The loop half-width at the footpoints is chosen as $2.5 \mathrm{Mm}$. Due to the absence of gravity the plasma $\beta=2 c_{\mathrm{s}}{ }^{2} /\left(\gamma V_{\mathrm{A}}{ }^{2}\right)$ grows from 0.012 at the loop footpoints to 0.054 at the loop apex in the ambient medium. This growth is not quite realistic, but cannot be avoided in the absence of gravity. Importantly, the value of $\beta$ is low everywhere in the loop.

\subsection{Perturbations}

As we are interested in impulsively excited waves, we launch a hot pulse in temperature or, equivalently, in the mass density and pressure at time $t=0$ from the location $x=z=0$, i.e. below the loop apex. This pulse has the spatial form:

$$
\begin{aligned}
& \frac{\delta \varrho(x, z, t=0)}{\varrho_{0}}=A_{\varrho} \mathrm{e}^{-\left(x^{2}+z^{2}\right) / w^{2}}, \\
& \frac{\delta p(x, z, t=0)}{p_{0}}=A_{\mathrm{p}} \mathrm{e}^{-\left(x^{2}+z^{2}\right) / w^{2}},
\end{aligned}
$$

where $w=35 \mathrm{Mm}$ is the initial pulse width. We choose initial relative amplitudes of the pulse $A_{\varrho}=1.5$ and $A_{\mathrm{p}}=15$, which corresponds to a pulse that is 6.4 times hotter than the corona.

It is noteworthy here that the observed relative dimension of the flaring site $w$ with respect to the loop size is rather large (e.g., Nakariakov et al. 2004). The initial location of the pulse corresponds to the flaring site. Unfortunately, the observations do not indicate directly the location of the flaring site (Wang \& Solanki 2004). However, we expect that in order to produce a vertical oscillation of a loop the flare site must be located close to the plane containing the loop. Thus it is well suited to be modelled in 2-D. The adopted pulse parameters were chosen such that they lead to numerical results which show encouraging agreement with observations.

\subsection{Numerics}

We use the Versatile Advection Code (VAC) developed by Tóth (1996). This code provides a choice of different numerical schemes to advance a system of hyperbolic equations in 1, 2 or 3D. Here we use the Flux Corrected Transport scheme (Boris \& Book 1973) to solve the MHD equations in 2D. Numerical tests obtained with VAC can be found in Tóth \& Odstrčil (1996).

In multi-dimensional MHD simulations the numerical value of the divergence of the magnetic field can become different from zero. There are several options in VAC to fix this problem. Here we use the projection scheme, which eliminates the divergence of the magnetic field after each time step by solving a Poisson equation iteratively.

Equations (1)-(5) are solved numerically in an Eulerian box with the $x$ - and $z$-dimensions $(-L, L) \times(0,2 L)$. This box is covered by a grid of $600 \times 600$ numerical cells. Grid convergence studies based on grid refinement $(300 \times 300,400 \times 500$, $600 \times 600$ and $900 \times 900$ ) were performed to show that the numerical results are not affected by poor spatial resolution. We apply line-tying boundary conditions, with $\boldsymbol{V}=0$, at the bottom and at the left and right hand sides of the simulation region. Open boundary conditions, with zero-gradient extrapolation of all plasma variables, are adopted for the top boundary.

\section{Results}

Figure 1 displays time-signatures of the mass density of the loop apex (shown is a cut along the line $x=0$ ). The presence of two temporal scales of oscillation can be distinguished: (a) long period oscillations associated with global loop movement which is triggered by the initial pulse and (b) short period oscillations seen in mass density, although with a relatively low amplitude. The long period fluctuations are strongly damped fast kink oscillations. The short period oscillations represent magnetosonic waves which are trapped by the loop cavity. The general resemblance of Fig. 1 to the observational data of Wang \& Solanki (2004) is striking, although there are differences in detail. Similarities include the strong damping, and the depletion of mass density at times of maximum expansion. The loop system moved outward by a maximum distance of about $4000 \mathrm{~m}$ with an initial velocity of $V_{z}=22 \mathrm{~km} \mathrm{~s}^{-1}$, which are smaller than the observational values of $7900 \mathrm{~m}$ and $130 \mathrm{~km} \mathrm{~s}^{-1}$ (Wang \& Solanki 2004). The initial pulse in the simulations is obviously weaker than the one giving rise to the observed oscillations. We found, however, that if we increase the strength of the initial pulse by a factor of two, the loop becomes unstable. The relatively low stability of the loop in our simulations may have to do with the neglect of gravity (plasma $\beta$ increasing with height) or with the simple magnetic field topology applied. The seeming filamentation of the loop seen in Fig. 1 may be a numerical artifact which is an inherent feature of the Flux Corrected Transport (FCT) method (Boris $\&$ Book 1973) that was used in the VAC code. This feature was not observed with the second-order TVD Lax-Friedrich method implemented in the code.

Figure 2 shows difference images of mass density evaluated at two times corresponding to consecutive peaks in the displacements (see Fig. 1). White (black) shading denotes an excess (depletion) of mass density in comparison to the earlier moment of time. Note that the loop is uniformly displaced and the maximum density displacement occurs near the loop apex. These profiles reveal that the first kink mode is excited, in agreement with the observations (Wang \& Solanki 2004, their Figs. 3c,d).

The global oscillations of the loop can be traced by following the position of the loop apex in time (Fig. 3). The apex position is estimated as the maximum of the Gaussian function

$G(z)=G_{0} \cdot \exp \left[-\frac{\left(z-G_{1}\right)^{2}}{2 G_{2}^{2}}\right]+G_{3}$

fitted across the loop. Here $G_{1}$ denotes the position of the loop apex, $G_{0}+G_{3}$ is the amplitude of Gaussian function (density at the loop apex) and $G_{2}$ is the half-width of the Gaussian function (half-width of the loop). 


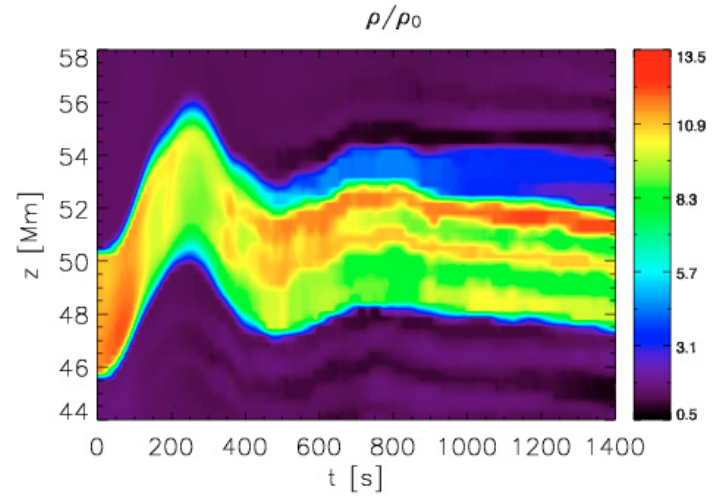

Fig. 1. Time-signature of the mass density (colour) at the apex of the loop.
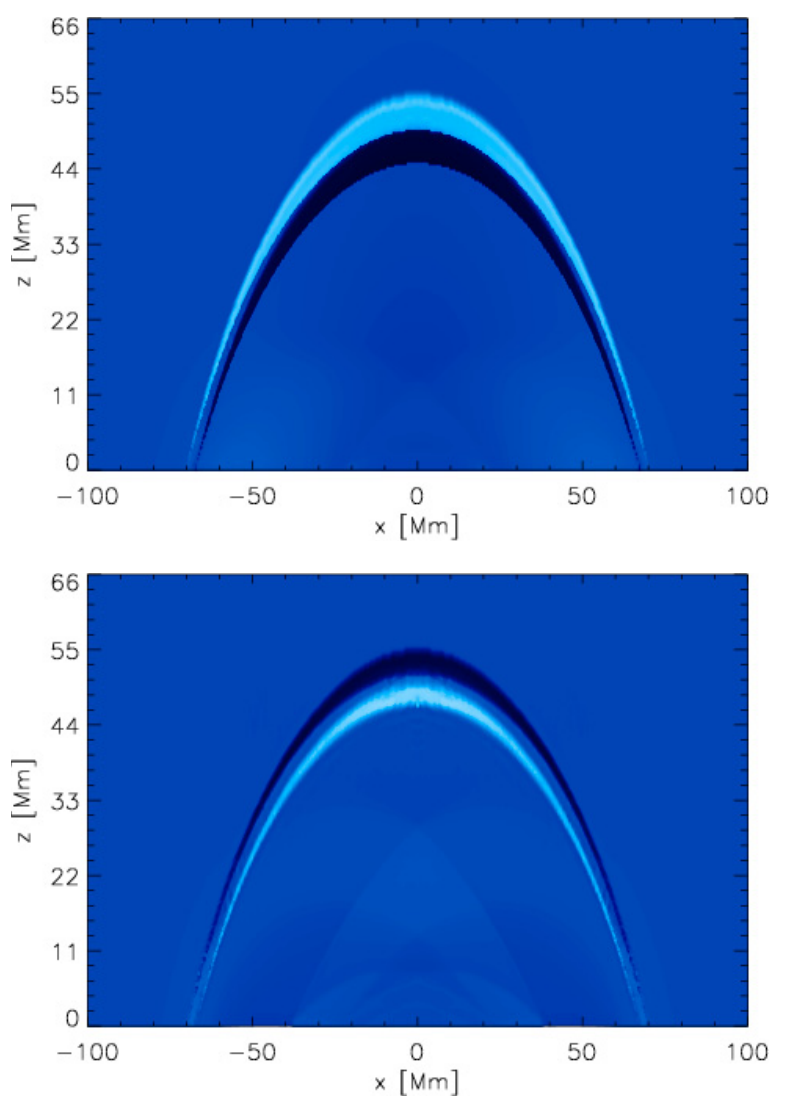

Fig. 2. Difference images of mass density spatial profiles evaluated over two different intervals of time: $\varrho(t=240 \mathrm{~s})-\varrho(t=0)$ (upper panel $)$ and $\varrho(t=460 \mathrm{~s})-\varrho(t=240 \mathrm{~s})$ (lower panel $)$.

We get a wave period and damping time of the oscillation by fitting the apex position vs. time (Fig. 3) to the damped sine function

$D(t)=D_{0}+D_{1} \cdot \sin \left(D_{2} t+D_{3}\right) \exp \left(-D_{4} t\right)$,

where $P=2 \pi / D_{2}$ denotes the period of the oscillation and $\tau=1 / D_{4}$ is the damping time. The above fitting provides $P=496 \mathrm{~s}$ and $\tau=223 \mathrm{~s}$. This period is about twice larger than the observed $\sim 230 \mathrm{~s}$ period of the displacement oscillations (Wang \& Solanki 2004), while the damping time is approximately three times shorter than the observed value of 11.9 min (Wang \& Solanki 2004). The difference in the
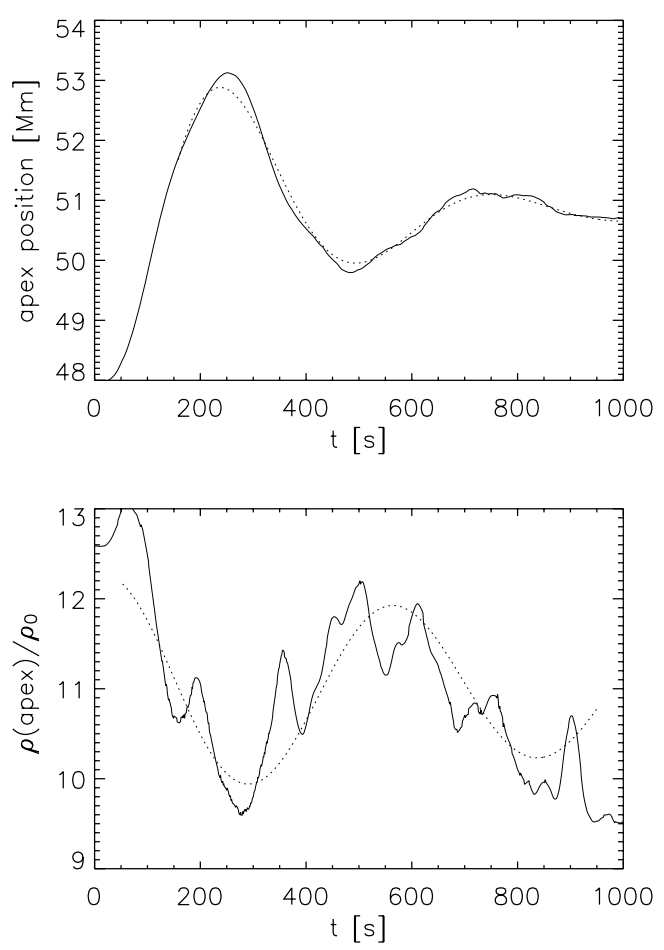

Fig. 3. Solid lines denote the position of the loop apex a) and mass density at the loop apex b) vs. time. Dotted lines correspond to damped sine functions fitted to the solid lines.

period is not a serious drawback of the model, since by increasing $V_{\mathrm{A} 0}$ by a factor of about two we would obtain the observed period. However, irrespective of the choice of $V_{\mathrm{A} 0}$, the ratio of the damping time to the wave period, $\tau / P$, reaches a value of 0.5 for the numerical data, while for the observation $\tau / P=3$. This difference may result from the simple physical model we adopted. The identification of the cause of the strong wave damping is not straightforward, although wave leakage, possibly enhanced by the curvature of the loop may play a role (Ruderman \& Roberts 2002; Van Doorsselaere et al. 2004; Andries et al. 2005). Figure 4, which shows vertical flow component across the apex of the loop reveals a significant amount of fast magnetosonic waves. There could potentially be leaking energy to the ambient plasma, although considerable farther simulations and analysis are required to identify the true cause of the damping and why it is stronger in the simulations than in the observations.

A comparison between Figs. $3 a$ and $3 b$ reveals that whereas the loop apex shows a smooth oscillation that is well fit by a damped sine function, the mass density, in addition to showing an oscillation with the same period and damping time, also exhibits high frequency oscillations. After removing the longer term variations we determine a period of $150 \mathrm{~s}$ for the higher frequency component. The fact that these short periods are not visible in Fig. 3a suggests that the short period pulsations do not correspond to higher harmonic kink modes.

It is noteworthy that, unlike in the analyzed observational data of Wang \& Solanki (2004), the simulated loop does not return to its original position at large times, but retains an offset to higher $z$. As this feature is present for all numerical methods and resolutions that we have tried, we conclude that the offset 


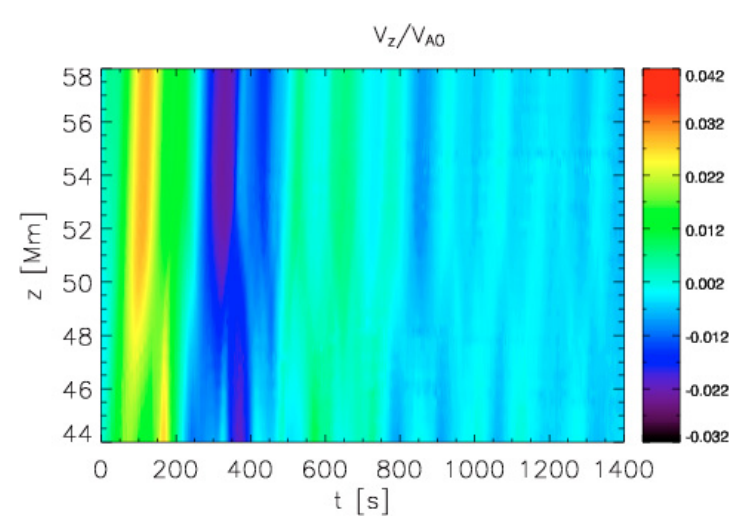

Fig. 4. Time-signature of the vertical component of the flow for the same cut as shown in Fig. 1.

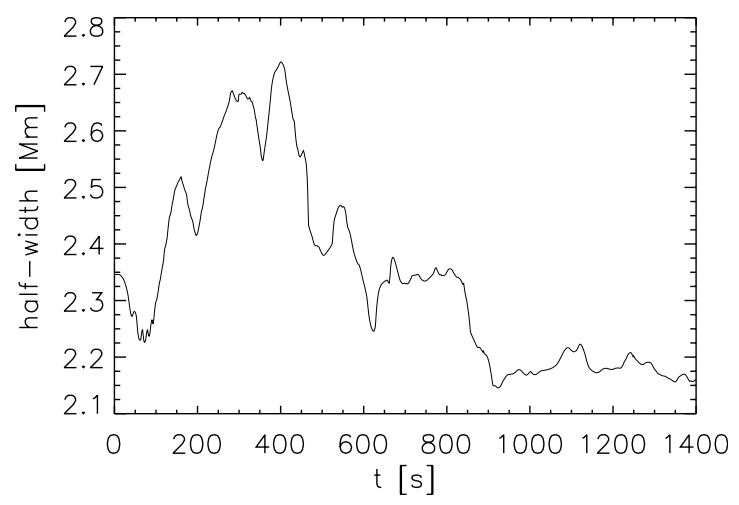

Fig. 5. Half-width of the loop at the apex vs. time.

reflects a new equilibrium the loop attains after being perturbed by the initial pulse. This offset can also be a result of the added energy/mass under the loop by the pulse (17) which prevents it from settling the same equilibrium height.

In Fig. $3 b$ it is discernible that the loop is rarified when it is largest, while it is denser in its most compact state. Using $I \sim$ $\varrho^{2}$, where $I$ denotes intensity, this implies that the loop is darker and brighter when it has expanded and compressed, again in good agreement with the observations (Wang \& Solanki 2004). Note that the phase shift between position and density is not exactly $180^{\circ}$ (as determined from the dotted curves in Fig. 3). At the end of the simulation the loop is clearly less dense than at the beginning. This loop evacuation is due to the energy leakage. During this process plasma flows into the ambient medium, smoothing initially sharp loop edges and evacuating its interior. As we have verified that numerical diffusion is negligibly small, this process is not an inherent numerical artifact.

The oscillation is thus not a pure kink mode, but shows some compressibility. The initial pulse excites a packet of waves in which kink waves have the highest contribution but compressible waves like fast magnetosonic sausage or slow waves are present too. This conclusion is supported by Fig. 5, which displays the half-width of the loop at the apex vs. time. The dominant feature of the figure is the broadening of the loop when the pulse hits it, followed by a slow relaxation to roughly its original width. In addition, a shorter period oscillation of the half-width is also visible. This oscillation, with a period of $130-140 \mathrm{~s}$, is more clearly seen in Fig. 6, where the half-width

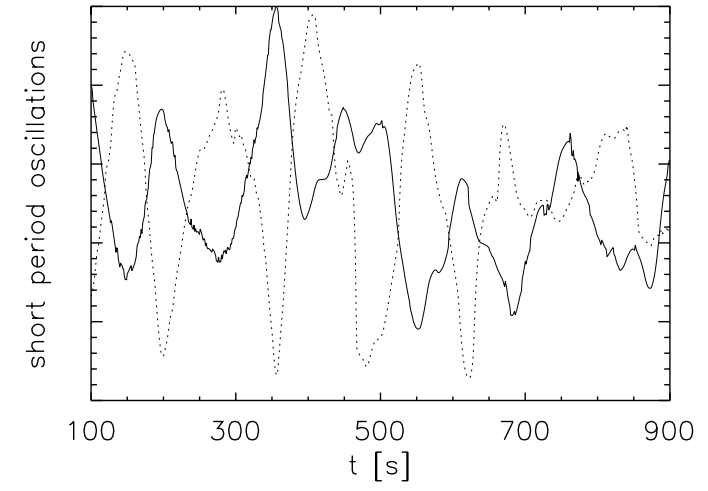

Fig. 6. Short period oscillations of mass density at the loop apex (solid line) and half-width of the loop (dashed line) at the apex vs. time. These oscillations are obtained from Figs. $3 b$ and 5 by filtering out long period oscillations.

has been plotted after removing the longer term changes. The similarly filtered density at the loop apex is also plotted. For the density profile the dotted curve in Fig. 3b, described by Eq. (19), was first removed.

Figure 6 clearly shows the anti-correlation between halfwidth and density. This antiphase behavior is consistent with the expectations for a fast sausage mode. It is noteworthy here that a sausage mode can propagate freely only for wave periods $\tau$ shorter than the cut-off period $\tau_{\mathrm{c}}$ that is given by (Edwin \& Roberts 1982, 1983)

$$
\tau_{\mathrm{c}}=\frac{4 a \sqrt{V_{\mathrm{Ae}}^{2}-V_{\mathrm{A}}^{2}}}{V_{\mathrm{Ae}} V_{\mathrm{A}}}=\frac{4 a \sqrt{1-\varrho_{0} / \varrho_{\mathrm{i}}}}{V_{\mathrm{A}}},
$$

where $a$ is the half-width of a slab. We get $\tau_{\mathrm{c}}=8.56 \mathrm{~s}$ for $a=2.5 \mathrm{Mm}$ at the loop foot-points and $\tau_{\mathrm{c}}=13.70 \mathrm{~s}$ for a maximum value of $a=4 \mathrm{Mm}$ at the apex. Since the wave period is much larger than both these estimates, the sausage wave seen in our simulations cannot be purely guided along the loop. The excited wave is therefore a leaky sausage wave (Edwin \& Roberts 1983). It is probably leakage from these waves which leads to the fast modes seen near the loop apex of Fig. 4.

\section{Summary}

In this paper we have studied numerically the impulsive excitation of oscillations of a highly magnetised solar coronal loop that is embedded in a two-dimensional magnetic arcade. In the applied model we have neglected several important factors such as: the three-dimensional geometry of loops and the flare site, radiative losses, and gravitational stratification. A 2$\mathrm{D}$ model may be more apropriate for the excitation of vertical oscillations than of other transverse oscillation modes, since the source of the initial pulse must be located in or near the loop plane. Of particular interest is the influence of gravity, which will be studied in a future investigation. Among loop oscillations two scales were distinguished: (a) long period (496 s) oscillations were interpreted as standing fast kink modes and (b) short period (140 s) oscillations we associate with leaky sausage waves. The observational signature of the kink wave is similar to the actual TRACE observations reported 
by Wang \& Solanki (2004), showing rapid damping and an anti-correlation between displacement and density. Differences in amplitude and period are probably due to differences in the strength of the initial pulse, the Alfvén speed, and loop length between modeled and the observed loop. The short period sausage mode, although so far not observed, may play a role in explaining the damping of the kink mode. It would be of great interest to look for such waves in conjunction with transverse loop oscillations.

The conjecture made here regarding the damping of the excited waves is that it is due to leakage. However, it cannot be explained fully on the basis of our present arguments. The issue of damping of loop oscillations is a rather intricate one and requires farther studies.

Acknowledgements. K.M. expresses his sincere thanks to Dr. Luigi Nocera for stimulating discussions. M.S. thanks Dr. Gherardo Valori for his helpful comments on running the VAC code which is provided at http://www.phys.uu.nl/ toth/. G.T. acknowlegdes support from the Hungarian Science Fundation (OTKA, grant No. T047042). The authors thank the referee for his/her stimulating comments. M.S.'s \& K.M.'s work was financially supported by a grant from the State Committee for Scientific Research Republic of Poland, with KBN grant No. 2 PO3D 01625.

\section{References}

Andries, J., Goossens, M., Hollweg, J. V., Arregui, I., \& Van Doorsselaere, T. 2005, A\&A, 430, 1109

Aschwanden, M., Fletcher, L., Schrijver, C., \& Alexander, D. 1999, ApJ, 520, 880

Aschwanden, M. 2002, in Proc. IAU Colloq., 188

Boris, J. P., \& Book, D. L. 1973, J. Comput. Phys., 11, 38, ESA SP-505, Noordwijk, 191
Cooper, F. C., Nakariakov, V. M., \& Williams, D. R. 2003, A\&A, 409, 325

Edwin, P. M., \& Roberts, B. 1982, Sol. Phys., 76, 239

Edwin, P. M., \& Roberts, B. 1983, Sol. Phys., 88, 179

del Zanna, L., Schaekens, E., \& Velli, M. 2005, A\&A, 431, 1095

Klimchuk, J. A., Tanner, S. E. M., \& De Moortel, I. 2004, ApJ, 616, 1232

Murawski, K., Selwa, M., \& Rossmanith, J. A. 2005a, Sol. Phys., submitted

Murawski, K., Selwa, M., \& Nocera, L. 2005b, A\&A, in press

Nakariakov, V. M. 2003, in The Dynamic Sun, ed. B. Dwivedi (CUP)

Nakariakov, V. M., \& Ofman, L. 2001, A\&A, 372, L53

Nakariakov, V. M., Arber, T. D., Ault, C. E., et al. 2004, MNRAS, 349, 705

Oliver, R., Murawski, K., \& Ballester, J. L. 1998, A\&A, 330, 726

Roberts, B. 2000, Sol. Phys., 193, 139

Roberts, B. 2002, in Solar variability: from core to outer frontiers, The 10th European Solar Physics Meeting, 9-14 September 2002, Prague, Czech Republic, ed. A. Wilson, ESA SP-506, 2 (Noordwijk: ESA Publications Division), ISBN 92-9092-816-6, 481

Ruderman, M. S., \& Roberts, B. 2002, ApJ, 577, 475

Selwa, M., Murawski, K., \& Solanki, S. K. 2005, A\&A, in press

Shergelashvili, B. M., Zaqarashvili, T. V., Poedts, S., \& Roberts, B. 2005, A\&A, 429, 767

Terradas, J., Oliver, R., \& Ballester, J. L. 2005, ApJ, 618, L149

Terradas, J., \& Ofman, L. 2004, ApJ, 610, 523

Torrence, C., \& Compo, G. P. 1998, Bull. Amer. Meteor. Soc., 79, 61

Tóth, G. 1996, Astrophys. Lett. Comm., 34, 245

Tóth, G., \& Odstrčil, D. 1996, J. Comput. Phys., 128, 82

Van Doorsselaere, T., Andries, J., Poedts, S., \& Goossens, M. 2004, ApJ, 606, 1223

Wang, T. J., \& Solanki, S. K. 2004, A\&A, 421, L33

Wang, T. J., Solanki, S. K., Curdt, W., Innes, D. E., \& Dammash, I. E. 2002, ApJ, 574, L101 